05,11

\title{
Аномалии магнитной восприимчивости при фазовых переходах второго рода вне точки Кюри
}

\author{
(C) Г.К. Чепурных ${ }^{1}$, В.А. Чёрная ${ }^{1}$, О.Г. Медведовская ${ }^{2}$ \\ ${ }^{1}$ Институт прикладной фризики, \\ Сумы, Украина \\ ${ }^{2}$ Сумский государственный педагогический университет им. А.С. Макаренко, \\ Сумы, Украина \\ E-mail: ipfmail@ipfcentr.sumy.ua
}

(Поступила в Редакцию 6 февраля 2018 г.)

\begin{abstract}
Учитывая неиссякаемый интерес к изучению особенностей физических свойств в окрестности фазовых переходов, а также рост экспериментальных исследований фторида кобальта, изучены особенности магнитной восприимчивости в окрестности критического поля $H_{\mathrm{C}}$, при котором фторид кобальта из антиферромагнитной фазы переходит в угловую в виде перехода второго рода. При этом обнаружено, что в магнитном поле $\mathbf{H} \| C_{4}$ магнитная восприимчивость обращается в бесконечность при $H \rightarrow H_{\mathrm{C}}$. Показано, что при отклонении направления магнитного поля от оси $C_{4}$ восприимчивость в критическом поле $H_{\mathrm{C}}$ оказывается конечной. Также показано, что изменение восприимчивости с изменением величины магнитного поля значительно уменьшается при крайне незначительном отклонении поля $\mathbf{H}$ от оси $C_{4}$. Поскольку расчеты выполнены в рамках теории фазовых переходов Ландау, то обращается внимание на сходство и отличие полученных результатов с результатами в окрестности точки Кюри, полученными также путем использования теории фазовых переходов Ландау.
\end{abstract}

DOI: $10.21883 /$ FTT.2018.09.46382.058

\section{1. Введение}

В настоящее время окрестность точки Кюри является единственным случаем, когда при фазовых переходах второго рода возникают аномалии магнитной воосприимчивости ${ }^{1}[1,2]$.

Исследованиям фазовых переходов посвящены монографии (см., например, $[4,5])$ и то, что фазовые переходы представляют одно из наиболее распространенных и интересных явлений, отмечено ${ }^{2}$ в [6-9].

Поскольку в последние годы увеличилось количество экспериментальных исследований [10-15], связанных с использованием фторида кобальта $\left(\mathrm{CoF}_{2}\right)$, то в работе [16] изучено состояние фторида кобальта в окрестности критического значения $H_{\mathrm{C}}$ продольного магнитного поля $\mathbf{H}$, при котором магнитная подсистема этого кристалла переходит в угловую фазу $[17,18]$ вместо обычно наблюдаемого перехода из состояния I $\|\mathbf{A}\|[001]$ в состояние $\mathbf{l} \perp \mathbf{A}(\mathbf{A}-$ ось легчайшего намагничивания, $\mathbf{l}$ - вектор антиферромагнетизма).

При этом обнаружено, что состояние магнитной подсистемы $\mathrm{CoF}_{2}$ при $H=H_{\mathrm{C}}$ оказалось крайне чувствительным к незначительному отклонению вектора магнитного поля Н от оси $C_{4}$, несмотря на необычайно значительную величину магнитной анизотропии $[17,18]$.

\footnotetext{
${ }^{1}$ Напомним, что при подходе к трикритической точке также возникают аномалии магнитной восприимчивости, однако трикритическая точка является точкой перехода линии фазовых переходов второго рода в линию переходов первого рода $[1,3]$.

2 В [6-9] приведены доклады, сделанные 21 декабря 2016 года на Научной сессии Отделения физических наук Российской академии наук „Старое и новое в физике фазовых переходов“.
}

Поскольку также выяснилось, что эта высокая чувствительность исчезает при изменении величины магнитного поля всего лишь на тысячные доли $H_{\mathrm{C}}$, то для этого случая возникает необходимость в обнаружении возможных аномалий магнитной восприимчивости.

Для выяснения сходства и отличия наших выводов (в рамках теории фазовых переходов Ландау) о поведении магнитной восприимчивости в окрестности поля $H_{\mathrm{C}}$ с выводами в окрестности точки Кюри $T_{\mathrm{C}}$, приведенными в $[1,2]$ (также в рамках теории Ландау), кратко напомним эти выводы.

На основании $[1,2]$ в окрестности $T_{\mathrm{C}}$ термодинамический потенциал $\phi(P, T, \eta)$ можно записать в виде

$$
\phi=\phi_{0}+A \eta^{2}+B \eta^{4}-\eta h V,
$$

где $A=a\left(T-T_{\mathrm{C}}\right), \eta-$ макроскопический магнитный момент (отнесенный к единице объема), $h$ - магнитное поле, $V$ - объем тела.

Благодаря учету возмущающего оператора вида $\eta h V$ использование (1) приводит к следующим выражениям $[1,2]$ для магнитной восприимчивости:

$$
\begin{gathered}
\chi=\left(\frac{\partial \eta}{\partial h}\right)_{h \rightarrow 0}=\frac{V}{4 a\left(T_{\mathrm{C}}-T\right)} \quad \text { при } \quad T<T_{\mathrm{C}}, \\
\chi=\left(\frac{\partial \eta}{\partial h}\right)_{h \rightarrow 0}=\frac{V}{2 a\left(T-T_{\mathrm{C}}\right)} \quad \text { при } \quad T>T_{\mathrm{C} .}
\end{gathered}
$$

Параметр порядка $\eta$ является однокомпонентной величиной и в приведенной работе [16] параметр порядка также является однокомпонентной величиной. 


\section{2. Выбор плотности энергии и составление уравнений}

Кристалл $\mathrm{CoF}_{2}$ исследуется в течение многих десятков лет и соответственно этому во многих работах записывается плотность энергии, используя которую, составляются уравнения, определяющие состояние магнитной подсистемы (см. ссылки, например, в [19]). В данной работе используется плотность энергии, предложенная в [18], и составленные там же уравнения состояния.

Так как изучаются особенности физических свойств в окрестности критического поля $H_{\mathrm{C}}$, то в плотности энергии $\mathscr{H}$, предложенной в [18], будем учитывать слагаемые наиболее важные вблизи $H_{\mathrm{C}}$ и поэтому

$$
\begin{aligned}
\mathscr{H}= & 2 M_{0}\left[\frac{1}{2} E \mathbf{m}^{2}+\frac{1}{2} G(\mathbf{m l})^{2}+D\left(m_{x} l_{y}+l_{x} m_{y}\right)\right. \\
& \left.+\frac{1}{2} A_{I}\left(l_{x}^{2}+l_{y}^{2}\right)-\mathbf{m H}\right],
\end{aligned}
$$

где $\mathbf{m}=\left(\mathbf{M}_{\mathbf{1}}+\mathbf{M}_{\mathbf{2}}\right) / 2 M_{0}, \quad \mathbf{I}=\left(\mathbf{M}_{\mathbf{1}}-\mathbf{M}_{\mathbf{2}}\right) / 2 M_{0}, \quad \mathbf{M}_{\mathbf{1}}$ и $\mathbf{M}_{2}$ - намагниченности подрешеток, $E$ и $G$ константы обменного взаимодействия, $D-$ константа взаимодействия Дзялошинского-Мория, $A_{I}$ - константа одноосной анизотропии, $H-$ внешнее магнитное поле.

Из-за зависимости намагниченности подрешеток $\mathbf{M}_{\mathbf{1}}$ и $\mathbf{M}_{2}$ от магнитного поля условие $\mathbf{m} \cdot \mathbf{l}=0$ невыполнимо.

Из необходимых условий существования минимума $\mathscr{H}$ как функции переменных $\theta, \varphi, \mathbf{m}(\theta$ и $\varphi-$ соответственно полярный и азимутальный углы вектора $\mathbf{l})$ следуют уравнения

$$
\partial \mathscr{H} / \partial \theta=0, \quad \partial \mathscr{H} / \partial \varphi=0, \quad \partial \mathscr{H} / \partial \mathbf{m}=0 .
$$

Определяя $\mathbf{m}$ из уравнения $\partial \mathscr{H} / \partial \mathbf{m}=0$ и, таким образом, исключая $\mathbf{m}$ из плотности энергии, в [18] записана плотность энергии как функция переменных $\theta$ и $\varphi$ и для этих переменных записаны также уравнения. В нашем случае все это имеет вид

$$
\begin{aligned}
\mathscr{H} & =2 M_{0}\left\{-\frac{1}{2 E}\left[H_{x}^{2}+H_{z}^{2}-2 H_{x} D \sin \theta \sin \varphi+D^{2} \sin ^{2} \theta\right]\right. \\
& +\frac{G}{2 E(E+G)}\left[H_{x}^{2} \sin ^{2} \theta \cos ^{2} \varphi+H_{x} H_{z} \sin 2 \theta \cos \varphi\right. \\
& +H_{z}^{2} \cos ^{2} \theta-2 H_{x} D \sin ^{3} \theta \sin 2 \varphi \cos \varphi-2 H_{z} D \\
& \left.\left.\times \sin ^{2} \theta \cos \theta \sin 2 \varphi+D^{2} \sin ^{4} \theta \sin ^{2} 2 \varphi\right]+\frac{1}{2} A_{1} \sin ^{2} \theta\right\} .
\end{aligned}
$$$$
\frac{\partial \mathscr{H}}{\partial \varphi}=\frac{1}{E} H_{x} D \sin \theta \cos \varphi
$$$$
+\frac{G}{2 E(E+G)}\left[-H_{x}^{2} \sin ^{2} \theta \sin 2 \varphi-H_{x} H_{z} \sin 2 \theta \sin \varphi\right.
$$$$
-4 H_{x} D \sin ^{3} \theta \cos 2 \varphi \cos \varphi+4 H_{x} D \sin ^{3} \theta \sin ^{2} \varphi \cos \varphi
$$$$
\left.-4 H_{z} D \sin ^{2} \theta \cos \theta \cos 2 \varphi+4 D^{2} \sin ^{4} \theta \sin 2 \varphi \cos 2 \varphi\right] \text {. }
$$

$\frac{\partial \mathscr{H}}{\partial \theta}=\frac{1}{E} H_{x} D \cos \theta \sin \varphi-\frac{1}{2 E} D^{2} \sin 2 \theta$

$+\frac{G}{2 E(E+G)}\left[H_{x}^{2} \sin 2 \theta \cos ^{2} \varphi+2 H_{x} H_{z} \cos 2 \theta \cos \varphi\right.$

$-H_{z}^{2} \sin 2 \theta-3 H_{x} D \sin 2 \theta \sin \theta \sin 2 \varphi \cos \varphi$

$-2 H_{z} D \sin 2 \theta \cos 2 \theta \sin 2 \varphi-+2 H_{z} D \sin ^{3} \theta \cos \theta \sin 2 \varphi$

$\left.+2 D^{2} \sin 2 \theta \sin ^{2} \theta \sin 2 \varphi\right]+\frac{1}{2} A_{1} \sin 2 \theta$.

Магнитное поле $\mathbf{H}$ находится в плоскости $Z X$. Магнитную восприимчивость в окрестности $H_{\mathrm{C}}$ будем определять при $\mathbf{H} \| C_{4}$ и при значениях угла $\psi$ между $\mathbf{H}$ и $C_{4}$ в пределах $0<\psi \leq 1^{\circ}$, так как в этих случаях изменение восприимчивости оказывается наиболее значительным.

\section{3. Восприимчивость в поле $\mathrm{H} \| \mathrm{C}_{4}$}

После разложения тригонометрических функций в ряд при $\theta \ll 1$ плотность энергии (5) согласно [16] при $\varphi=\pi / 4$ имеет вид:

$$
\begin{gathered}
\mathscr{H}=2 M_{0}\left(-\frac{H^{2}}{2 E}+A \theta^{2}+B \theta^{4}\right), \\
A=\frac{G}{2 E(E+G)}\left[-\left(H^{2}-H_{\mathrm{C}}^{2}\right)-2 D\left(H-H_{\mathrm{C}}\right)\right], \\
B=\frac{G}{6 E(E+G)}\left[H^{2}-H_{\mathrm{C}}^{2}+D\left(5 H-2 H_{\mathrm{C}}\right)+3 D^{2}\right] .
\end{gathered}
$$

Формулу (9) удобнее записать в виде

$$
A=\frac{G}{2 E(E+G)}\left[-\left(H-H_{\mathrm{C}}\right)\left(H+H_{\mathrm{C}}+2 D\right)\right] .
$$

В выражении (8) параметром порядка являетсяя угол $\theta$. Поэтому в согласии с $[1,2]$ из уравнения $\partial \mathscr{H} / \partial \theta=0$ следует

$$
\theta^{2}=-\frac{A}{2 B}
$$

Поскольку $H-H_{\mathrm{C}} \ll H_{\mathrm{C}}$, то выражения для $A$ и $B$ можно записать в виде

$$
A=-\frac{G\left(H_{\mathrm{C}}+D\right)}{E(E+G)}\left(H-H_{\mathrm{C}}\right), \quad B=\frac{D G\left(7 H_{\mathrm{C}}+3 D\right)}{6 E(E+G)} .
$$

Поэтому параметр порядка $\theta$ определяется формулой

$$
\theta=\sqrt{\frac{3\left(H_{\mathrm{C}}+D\right)}{D\left(7 H_{\mathrm{C}}+3 D\right)}} \times \sqrt{H-H_{\mathrm{C}}} .
$$

Для определения компонент тензора статической магнитной восприимчивости, выражения для намагниченности, определенные в [18], при условиях $H-H_{\mathrm{C}} \ll H_{\mathrm{C}}$, $\theta \ll 1$, и $G=E$ можно записать в виде

$$
\begin{gathered}
m_{x}=m_{y}=-\frac{\sqrt{2}}{4} \frac{H_{\mathrm{C}}+D}{E} \theta, \\
m_{z}=\frac{1}{2 E}\left[H+\frac{3\left(H-H_{\mathrm{C}}\right)\left(H_{\mathrm{C}}+D\right)}{7 H_{\mathrm{C}}+3 D}\right] .
\end{gathered}
$$


Рассчитанные значения магнитной восприимчивости $\chi_{x z} \sqrt{2}\left(E / H_{\mathrm{C}}\right)$, изменения $\Delta \theta$ угла $\theta$, обусловленные изменением магнитного

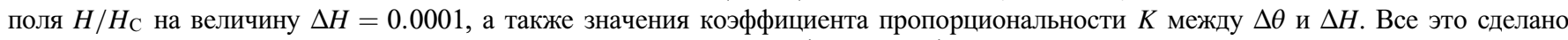
для случая, когда угол $\psi$ между $\mathbf{H}$ и $C_{4}$ находится в пределах $0.25^{\prime} / \leq \psi \leq 1^{\prime}$.

\begin{tabular}{|c|c|c|c|c|c|c|c|}
\hline \multicolumn{8}{|c|}{$\psi=0.25^{\prime}$} \\
\hline $\begin{array}{c}H / H_{\mathrm{C}} \\
\theta(\mathrm{deg}) \\
\Delta \theta(\mathrm{rad}) \\
K \\
\text { Восприимчивость }\end{array}$ & $\begin{array}{c}0.997 \\
0.5785 \\
0.0195 \\
195 \\
143.6\end{array}$ & $\begin{array}{c}0.998 \\
0.8533 \\
0.0413 \\
413 \\
304\end{array}$ & $\begin{array}{c}0.999 \\
1.5057 \\
0.1054 \\
1054 \\
775.9\end{array}$ & $\begin{array}{c}1 \\
2.8911 \\
0.1587 \\
1587 \\
1168\end{array}$ & $\begin{array}{c}1.001 \\
4.3907 \\
0.1356 \\
1356 \\
995\end{array}$ & $\begin{array}{c}1.002 \\
5.6415 \\
0.1134 \\
1134 \\
829.9\end{array}$ & $\begin{array}{c}1.003 \\
6.7016 \\
0.0981 \\
981 \\
712.8\end{array}$ \\
\hline \multicolumn{8}{|c|}{$\psi=0.5^{\prime}$} \\
\hline $\begin{array}{c}H / H_{\mathrm{C}} \\
\theta(\mathrm{deg}) \\
\Delta \theta(\mathrm{rad}) \\
K \\
\text { Восприимчивость }\end{array}$ & $\begin{array}{c}0.997 \\
1.1312 \\
0.0356 \\
356 \\
262.1\end{array}$ & $\begin{array}{c}0.998 \\
1.6018 \\
0.0647 \\
647 \\
476\end{array}$ & $\begin{array}{c}0.999 \\
2.4443 \\
0.1082 \\
1082 \\
795.6\end{array}$ & $\begin{array}{c}1 \\
3.6411 \\
0.126 \\
1260 \\
926\end{array}$ & $\begin{array}{c}1.001 \\
4.8669 \\
0.1162 \\
1162 \\
851.9\end{array}$ & $\begin{array}{c}1.002 \\
5.9681 \\
0.1029 \\
1029 \\
752\end{array}$ & $\begin{array}{c}1.003 \\
6.9453 \\
0.0919 \\
919 \\
667.1\end{array}$ \\
\hline \multicolumn{8}{|c|}{$\psi=1^{\prime}$} \\
\hline $\begin{array}{c}H / H_{\mathrm{C}} \\
\theta(\mathrm{deg}) \\
\Delta \theta(\mathrm{rad}) \\
K \\
\text { Восприимчивость }\end{array}$ & $\begin{array}{c}0.997 \\
2.1051 \\
0.0542 \\
542 \\
398.7\end{array}$ & $\begin{array}{c}0.998 \\
2.7464 \\
0.0766 \\
766 \\
563\end{array}$ & $\begin{array}{c}0.999 \\
3.6029 \\
0.0947 \\
947 \\
694.8\end{array}$ & $\begin{array}{c}1 \\
4.5845 \\
0.1001 \\
1001 \\
732.7\end{array}$ & $\begin{array}{c}1.001 \\
5.5722 \\
0.0963 \\
963 \\
702.7\end{array}$ & $\begin{array}{c}1.002 \\
6.5055 \\
0.0896 \\
896 \\
651.6\end{array}$ & $\begin{array}{c}1.003 \\
7.3708 \\
0.0830 \\
830 \\
601.4\end{array}$ \\
\hline
\end{tabular}

Используя (11a), (12), находим

$$
\chi_{x z}=\frac{\partial m_{x}}{\partial H_{z}}=\chi_{y z}=\frac{\partial m_{y}}{\partial H_{z}}=\frac{a}{\sqrt{H-H_{\mathrm{C}}}},
$$

где

$$
a=\sqrt{\frac{3\left(H_{\mathrm{C}}+D\right)}{D\left(7 H_{\mathrm{C}}+D\right)}} .
$$

Используя (13), получаем

$$
\chi_{z z}=\frac{\partial m_{z}}{\partial H_{z}}=\frac{1}{2 E}\left[1+\frac{3\left(H_{\mathrm{C}}+D\right)}{7 H_{\mathrm{C}}+3 D}\right] .
$$

Приведенные компоненты тензора восприимчивости определены для угловой фазы.

В антиферромагнитной фазе (I || A)

$$
\chi_{x z}=\chi_{y z}=0, \quad \chi_{z z}=\frac{1}{2 E} .
$$

Сравнивая полученное выражение (14) для восприимчивости с известными выражениями (2) для восприимчивости в окрестности точки Кюри, мы видим, что если в несимметричной фазе $\left(T<T_{\mathrm{C}}\right)$ восприимчивость обращается в бесконечность при $T \rightarrow T_{\mathrm{C}}$ благодаря учету возмущающего оператора $\eta h V$, то в нашем случае восприимчивости $\chi_{x z}, \chi_{y z}$ обращаются в бесконечность в несимметричной фазе при $H \rightarrow H_{\mathrm{C}}$ в „чистом“ виде, т.е. без учета возмущений.

Кроме того, если согласно [2] в несимметричной фазе $\left(T<T_{\mathrm{C}}\right)$ восприимчивость не является восприимчивостью в обычном смысле слова (т.е. коэффициентом пропорциональности между $M$ и $H)$, так как $M \neq 0$ и при $H=0$, то восприимчивость, определяемая соотношением (14), является восприимчивостью в обычном смысле слова.

Компонента $\chi_{z z}$ восприимчивости с ростом величины магнитного поля испытывает скачок в точке фазового перехода.

\section{4. Восприимчивость в наклонном магнитном поле}

Поскольку угол $\psi$ между Н и $C_{4}$ находится в пределах $0<\psi \leq 1^{\circ}$, то для определения восприимчивости $\chi_{x z}$, $\chi_{y z}$ воспользуемся уравнением

$$
\begin{aligned}
& \frac{1}{\sqrt{2}} \frac{H_{x}}{H_{\mathrm{C}}}\left[\left(1+\frac{E}{G}\right) \frac{D}{H_{\mathrm{C}}} \cos \theta+\frac{H_{z}}{H_{\mathrm{C}}} \cos 2 \theta\right] \\
& +\sin \theta\left\{\operatorname { c o s } \theta \left[-\frac{H_{z}^{2}}{H_{\mathrm{C}}^{2}}-2 \frac{H_{z} D}{H_{\mathrm{C}}^{2}} \cos \theta\right.\right. \\
& \left.\left.+2 \frac{D^{2}}{H_{\mathrm{C}}^{2}} \sin ^{2} \theta+1+2 \frac{D}{H_{\mathrm{C}}}\right]+\frac{H_{z} D}{H_{\mathrm{C}}^{2}} \sin ^{2} \theta\right\}=0
\end{aligned}
$$

и его численными решениями (см. рис. 1, 2), полученными в [16]. Учитывается, что $H_{x}=H \sin \psi=H \psi$, $H_{z}=H \cos \psi=H, D=50 \mathrm{kOe}, H_{\mathrm{C}}=210 \mathrm{kOе}$ и принимается условие $E=G$. 
Выражениями для намагниченностей $m_{x}, m_{y}$, определенных в [18], воспользуемся не в виде (12), а в виде ${ }^{3}$

$$
\begin{aligned}
& \sqrt{2}\left(E / H_{\mathrm{C}}\right) m_{x}=\sqrt{2}\left(E / H_{\mathrm{C}}\right) m_{y} \\
& =-\sin \theta\left[\frac{D}{H_{\mathrm{C}}}\left(1-\frac{G}{E+G} \sin ^{2} \theta\right)+\frac{H}{H_{\mathrm{C}}} \frac{G}{E+G} \cos \theta\right] .
\end{aligned}
$$

Восприимчивость будем определять в окрестности найденных численных решений, учитывая, что в окрестности поля $H_{\mathrm{C}}$ (как это наиболее четко следует из формулы 12) изменение намагниченности обусловлено главным образом изменением угла $\theta$.

Любое из решений, представленных на рис. 1, 2, будем обозначать через $\theta_{i}$, которое реализуется в магнитном поле $H_{i} / H_{\mathrm{C}}$ при заданных значениях угла $\psi$. Если при этом изменять магнитное поле на величину $\Delta H$, то при условии $\Delta H \ll H_{i} / H_{\mathrm{C}}$ изменение $\Delta \theta_{i}$ угла $\theta_{i}$ будет удовлетворять условию $\Delta \theta_{i} \ll \theta_{i}$. Из этого следует

$$
\Delta \theta_{i}=K_{i} \Delta H
$$

Полагая в соотношении (17) $\theta=\theta_{i}+\Delta \theta_{i}$ и учитывая формулу (18), намагниченность $m_{x i}$ в окрестности поля $H_{i} / H_{\mathrm{C}}$ запишем в виде

$$
\begin{aligned}
\sqrt{2}\left(E / H_{\mathrm{C}}\right) m_{x i} & =-\left\{\sin \theta_{i}+\cos \theta_{i}\right. \\
\times & {\left.\left[\frac{50}{210}\left(1-\frac{1}{2} \sin ^{2} \theta_{i}\right)+\frac{H_{i}}{2 H_{\mathrm{C}}} \cos \theta_{i}\right] K_{i} \Delta H\right\} . }
\end{aligned}
$$

Поэтому восприимчивость $\chi_{x z i}$ определяется соотношением

$$
\begin{aligned}
& \sqrt{2}\left(E / H_{\mathrm{C}}\right) \frac{\partial m_{x i}}{\partial(\Delta H)}=\sqrt{2}\left(E / H_{\mathrm{C}}\right) \chi_{x z i} \\
& =\cos \theta_{i}\left[\frac{50}{210}\left(1-\frac{1}{2} \sin ^{2} \theta_{i}\right)+\frac{H_{i}}{2 H_{\mathrm{C}}} \cos \theta_{i}\right] K_{i} .
\end{aligned}
$$

Затем, полагая в уравнении (16) $\theta=2.8911^{\circ}+\Delta \theta_{1}$, $\psi=0.25^{\prime}, \quad H / H_{\mathrm{C}}=1, \quad$ находим при ${ }^{4} \quad \Delta H=0.0001$, $\Delta \theta_{1}=0.1587$ и соответственно $K_{1}=1587$.

Восприимчивость $\chi_{x z i}$ определяется путем исследования соотношения (19a) при $\theta_{i}=\theta=2.8911^{\circ}$, $K_{i}=K_{1}=1587$.

Поэтому в окрестности заданного значения для угла $\theta$ и поля $H / H_{\mathrm{C}}$ при $\psi=0.25^{\prime}$ получаем ${ }^{5}$

$$
\left|\sqrt{2}\left(E / H_{\mathrm{C}}\right) \chi_{x z_{1}}\right|=1168 .
$$

\footnotetext{
${ }^{3}$ Поскольку $m_{x}=m_{y}$, то в дальнейшем будем писать только $m_{x}$ и соответственно только $\chi_{x z}$.

${ }^{4} \Delta H=0.0001$ будем использовать в окрестности всех решений, представленных на рис. 1,2.

5 Поскольку в наклонном магнитном поле основное внимание уделяется изменению магнитной восприимчивости $\chi_{x z}$, то записываем восприимчивость $\chi_{x z}$, умноженную на постоянную величину $\sqrt{2}\left(E / H_{\mathrm{C}}\right)$.
}

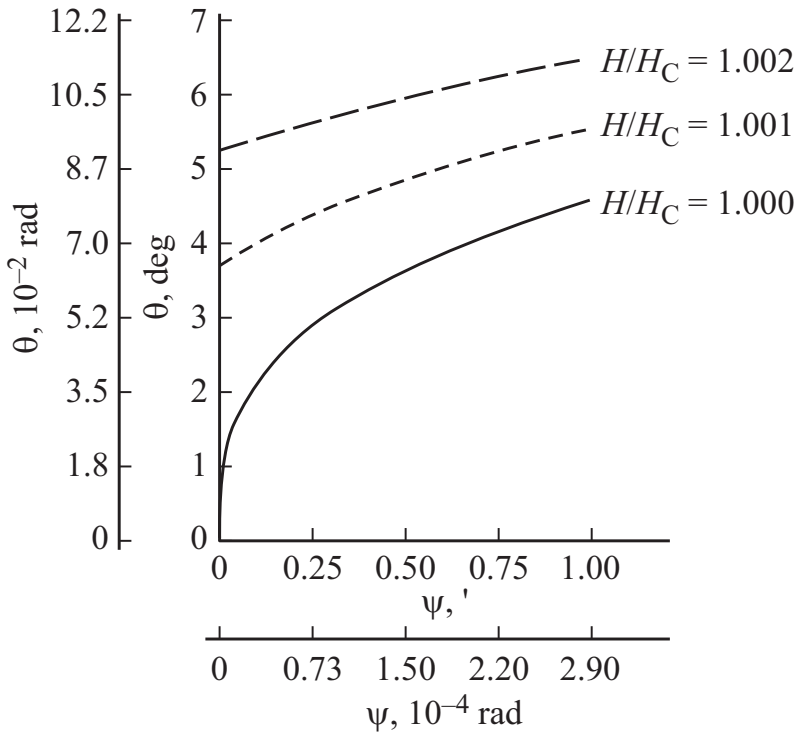

Рис. 1. Зависимость ориентации магнитной подсистемы кристалла $\mathrm{CoF}_{2}$ от ориентации магнитного поля $\mathbf{H}$ в полях $H / H_{\mathrm{C}} \leq 1: \psi-$ угол между осью $C_{4}$ и направлением поля $\mathbf{H}$, $\theta-$ угол между осью $C_{4}$ и ориентацией вектора антиферромагнетизма $\mathbf{l}$.

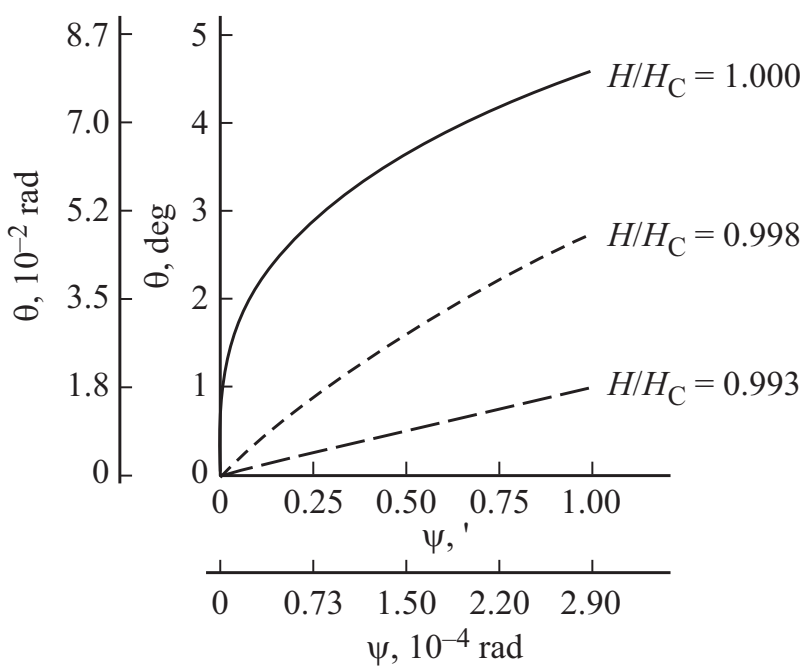

Рис. 2. Зависимость ориентации магнитной подсистемы кристалла $\mathrm{CoF}_{2}$ от ориентации магнитного поля Н в полях $H / H_{\mathrm{C}} \leq 1: \psi-$ угол между осью $C_{4}$ и направлением поля $\mathbf{H}$, $\theta-$ угол между осью $C_{4}$ и ориентацией вектора антиферромагнетизма $\mathbf{l}$.

Аналогичным способом определены все остальные значения магнитной восприимчивости в окрестности решений, представленных на рис. $1,2$.

Вычисленные значения магнитной восприимчивости в наклонном магнитном поле приведены в таблице и на рис. 3. Из этих данных следует, что при отклонении направления магнитного поля от оси $C_{4}$ восприимчивость $\chi_{x z}$ в критическом поле $H_{\mathrm{C}}$ оказывается конечной, тогда как в поле $\mathbf{H} \| C_{4}$ при $H \rightarrow H_{\mathrm{C}} \chi_{x z} \rightarrow \infty$. 


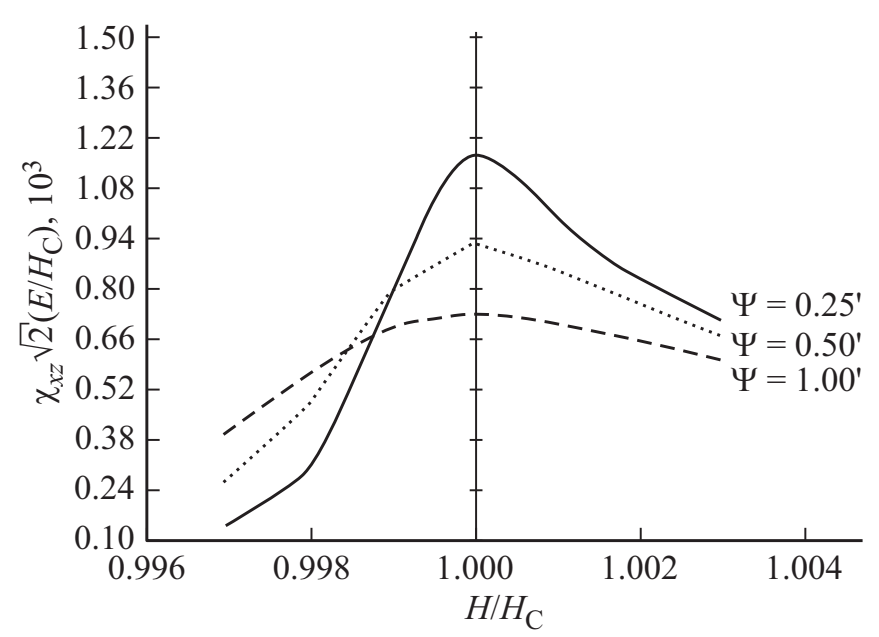

Рис. 3. Зависимость магнитной восприимчивости $\chi_{x z} \sqrt{2}\left(E / H_{\mathrm{C}}\right)$ от величины магнитного поля $H / H_{\mathrm{C}}$ при различных значениях угла $\psi$ между Н и $C_{4}$.

Из таблицы и рис. 3 также следует, что в окрестности критического поля $H_{\mathrm{C}}$ изменение восприимчивости $\chi_{x z}$ с изменением величины магнитного поля значительно уменьшается при крайне незначительном отклонении направления поля $H$ от оси $C_{4}$.

\section{5. Заключение}

Обнаруженная аномалия магнитной восприимчивости при фазовых переходах второго рода вне точки Кюри и ее изменения при отклонении направления магнитного поля от оси $C_{4}$ могут представлять интерес для дальнейших экспериментальных и теоретических исследований с целью обнаружения возможных особенностей физических свойств.

Нельзя исключать того, что некоторые из особенностей физических свойств в окрестности точки Кюри, на которые обращалось внимание в [1-9], могут проявляться в окрестности изучаемого нами фазового перехода второго рода. Это важно и потому, что материалы, в которых была обнаружена гигантская магнитострикция [20] $\left(\Delta l / l \sim 10^{-2}\right)$, если эти материалы подвергнуть более тщательному теоретическому изучению на предмет обнаружения возможных аномалий в уменьшении эффективной магнитной анизотропии при фазовых переходах второго рода, могут найти применение для создания высокочувствительных сенсоров и генераторов мощного звука.

Обратим внимание, что еще в работе [21] было доказано возникновение аномального поглощения звука вблизи точек фазового перехода второго рода не благодаря флуктуациям, а благодаря тому, что уже небольшое возмущение сильно меняет равновесное значение параметра порядка (см. в [1] с. 495, 499, 513).

Учитывая это обстоятельство, в работе [22] было предсказано аномально сильное возрастание безразмер- ного параметра связи спиновых и упругих волн в антиферромагнетиках при подходе к фазовому переходу.

\section{Список литературы}

[1] Л.Д. Ландау, Е.М. Лифшиц. Статистическая физика, часть I. Наука, Москва (1976).

[2] Л.Д. Ландау, Е.М. Лифшиц. Электродинамика сплошных сред. Наука, Москва (1982).

[3] А. Брус, Р. Каули. Структурные фазовые переходы. Москва (1984).

[4] Г. Стенли. Фазовые переходы и критические явления. Москва (1973).

[5] А.З. Паташинский, В.Л. Покровский. Флуктуационная теория фазовых переходов. Наука, Москва (1975).

[6] В.Н. Рыжков, Е.Е. Тареева, Ю.Д. Фомин, Е.Н. Циок. УФН 187, 9, 921 (2017).

[7] А.Н. Утюж, А.В. Михеенков. УФН 187, 9, 953 (2017).

[8] Е.И. Кац. УФН 187, 9, 1022 (2017).

[9] В.В. Бражкин. УФН 187, 9, 1028 (2017).

[10] T.R. Dugan, J.M. Goldberg, W.W. Brennessel, P.L. Holland. Organometallics 31, 4, 1349 (2012).

[11] Y.T. Teng, S.S. Pramana, J. Ding, T. Wu, R. Yazami. Electrochimica Acta 107, 301 (2013).

[12] M.J. Armstrong, A. Panneerselvam, C. O'Regan, M.A. Morrisab, J.D. Holmes. J. Mater. Chem. A 1, 10667 (2013).

[13] C.Y. Lee, Z. Su, K. Lee, H. Tsuchiya, P. Schmuki. Chem. Commun. 50, 7067 (2014).

[14] M.C. Leclerc, J.M. Bayne, G.M. Lee, S.I. Gorelsky, M. Vasiliu, I. Korobkov, D.J. Harrison, D.A. Dixon, R.T. Baker. J. Am. Chem. Soc. 137, 16064 (2015).

[15] J. Tan, L. Liu, S. Guo, H. Hu, Z. Yan, Q. Zhou, Z. Huang, H. Shu, X. Yang, X. Wang. Electrochimica Acta 168, 225 (2015).

[16] О.Г. Медведовская, Т.А. Федоренко, Г.К. Чепурных. ФТТ, 58, 2350 (2016).

[17] Н.Ф. Харченко, В.В. Еременко, Л.И. Белый. ЖЭТФ 82, 827 (1982).

[18] К.Г. Гуртовой, А.С. Лагутин, В.И. Ожогин. ЖЭТФ 83, 1941 (1982).

[19] Г.К. Чепурных. Области экстремальных характеристик магнитоупорядоченных кристаллов. Киев, Наукова думка (2010).

[20] К.П. Белов. СОЖ 3, 15 (1998).

[21] Л.Д. Ландау, И.М. Халатников. ДАН СССР 94, 469 (1954).

[22] С.В. Пелетминский. ЖЭТФ 37, 452 (1959).

Редактор Ю.Э. Китаев 